The Egyptian Journal of Hospital Medicine (April 2021) Vol. 83, Page 1404-1408

\title{
Incidence of Acute Kidney Injury in Hospitalized Patients with Coronavirus Disease 19 Infection
}

Walid Ahmed Ragab Abdelhamid

Internal Medicine Department, Faculty of Medicine, Zagazig University, Zagazig, Egypt

Corresponding author: Walid Ahmed Ragab Abdelhamid, Email: WAAbdelHamid@ medicine.zu.edu.eg, Tel. +201062904443

\begin{abstract}
Background: While the most important sequelae of coronavirus disease 2019 (COVID- 19) are alveolar injury and respiratory failure, other systems has also been involved, particularly the renal system. The occurrence of acute kidney injury (AKI) varies broadly among patients with COVID-19 infection in various studies. Nevertheless, there is a lack of information from Middle Eastern countries. Objective: To study the effects of COVID-19 infection on kidney function and determine the predictors of AKI in those patients. Patients and Methods: The research involved 66 patients with verified diagnosis of COVID-19 infection. They were classified into group 1 (55 subjects) who did not develop AKI and group 2 (15 subjects) who developed AKI. All study participants were subjected to detailed history taking, clinical data recording and serial laboratory investigations during the time of hospital admission.

Results: Both groups were sex-matched. Group 2 was significantly older than group 1 with a median age of 69 years and had lower diastolic blood pressure (DBP) than group 1. Group 2 had significantly higher acute inflammatory markers, higher serum creatinine, higher blood urea, lower serum total protein and lower estimated glomerular filtration rate (eGFR) at the time of presentation than group 1. Conclusion: AKI occurred in 22.7\% of patients with verified COVID-19 infection in this study. The significant predictors of AKI in these patients were lower DBP, higher C-reactive protein level, higher serum lactate dehydrogenase level, and lower baseline eGFR.

Keywords: COVID-19, Acute kidney injury, Glomerular filtration rate, Lactate Dehydrogenase, Cytokine Release Syndrome.
\end{abstract}

\section{INTRODUCTION}

In 2019, a highly infectious respiratory virus had spread widely in China and was officially nominated "coronavirus disease 19" by the World Health Organization ${ }^{(\mathbf{1})}$. The emerging coronavirus disease 19 (COVID-19) pandemic has become a global emergency because of its wide growth and high risk of death. The number of people diagnosed with COVID-19 is growing globally up to date. Patients with severe COVID-19 infection suffer from pneumonia and respiratory failure, then multiple organ dysfunction may eventually develop (2). Recent proofs have suggested that this virus can directly invade tissues or cause cytokine-mediated injury leading to hepatotoxicity, coagulopathy, or nephropathy (3). Additionally, chronic renal impairment was found to be directly linked to mortality in COVID-19 infection in descriptive studies in Spain, which involved over 20,000 patients ${ }^{(4)}$.

This research aimed to identify the impact of COVID19 infection on renal function and determine predictors of acute kidney injury (AKI) in COVID-19 infection.

\section{PATIENTS AND METHODS}

This research was a retrospective cohort study, which involved all verified COVID-19 adult patients who were admitted to the internal medicine department of the hospital from $1^{\text {st }}$ September 2020 to $31^{\text {st }}$ December 2020. If the participants had end-stage kidney disease or were kidney transplant recipients, they were excluded from the research. The subjects were categorized into two groups according to the occurrence of AKI. Group 1: It included 51 COVID-19 confirmed patients who did not develop AKI. They were 30 males and 21 females with ages ranging from 24 to 90 years with a median age of 52 years. Group 2: It included 15 COVID-19 confirmed patients who developed AKI during hospital admission. They were 8 males and 7 females with ages ranging from 19 to 86 years with a median age of 69 years.

All study participants were evaluated by recording of full history including associated comorbidities, physical examination and serial laboratory tests including renal function tests, serum calcium, serum phosphorus, serum alkaline phosphatase, complete blood count, serum lactate dehydrogenase, liver function test, D-dimer, serum troponin, serum ferritin and $\mathrm{C}$-reactive protein. Baseline eGFR was recorded from the last documented level within 1 year of hospitalization. COVID-19 infection was confirmed by testing for the virus using RT-PCR (Reverse transcription polymerase chain reaction). AKI was identified following the criteria of The Kidney Disease: Improving Global Outcome (KDIGO) for diagnosis of AKI ${ }^{(5)}$.

Statistical analysis: It was run by SPSS (Statistical Package for the Social Sciences) version 26. The Shapiro Walk test was utilized to assess normality of continuous 
data. Means of normally distributed continuous data were compared using independent sample Student's t-test, whereas Mann Whitney U test was applied for nonnormally distributed continuous data. Chi-square test was used to compare nominal data. Independent predictors of AKI were determined using forward logistic regression analysis. All variables found to be significant on univariate logistic regression with $\mathrm{P} \leq 0.05$ was considered for multiple regression analysis.

\section{Ethical approval:}

The Approval of the Institutional Review Board of the Ethical Committee of Zagazig university was obtained before the study. The study protocol followed the 1975 Helsinki declaration ethical guidelines. Participants shared in the study after taking informed written consent.

\section{RESULTS}

The study included 66 participants who were categorized into two groups. Demographic characteristics diverged significantly among the studied groups and had older age 2 group $(\mathrm{P}=0.028)$ and lower $\mathrm{DBP}(\mathrm{P}=$ 0.048 ) at the time of presentation than group 1 as demonstrated in table (1).

Table (1): Comparison between the two groups regarding demographic and clinical data

\begin{tabular}{|c|c|c|c|c|}
\hline Variable & Group 1 & Group 2 & Statistics & $P$ \\
\hline Age (years), Median (Range) & $52(24-90)$ & $69(19-86)$ & -2.196 & $0.028 *$ \\
\hline Male gender, No (\%) & $33(64.7 \%)$ & $8(53.3 \%)$ & 0.64 & 0.43 \\
\hline History of DM, No (\%) & $17(33.3 \%)$ & $9(60 \%)$ & 3.452 & 0.063 \\
\hline History of HTN, No (\%) & $21(41.2 \%)$ & $10(66.7 \%)$ & 3.024 & 0.082 \\
\hline History of CKD, No (\%) & $18(35.3 \%)$ & $9(60 \%)$ & 2.927 & 0.087 \\
\hline History of IHD, No (\%) & $10(19.6 \%)$ & $3(20 \%)$ & 0.001 & 0.97 \\
\hline SBP $(\mathrm{mm} \mathrm{Hg})$, Mean \pm SD & $132.2 \pm 17.6$ & $122.4 \pm 22.7$ & 1.77 & 0.08 \\
\hline DBP (mm Hg), Median (Range) & $80(53-100)$ & $70(30-90)$ & -1.981 & $0.048 *$ \\
\hline Pulse (beats per minute), Mean \pm SD & $94 \pm 21$ & $97 \pm 25$ & -0.44 & 0.66 \\
\hline Oxygen Saturation (\%), Median (Range) & $90(66-100)$ & $85(59-98)$ & -1.256 & 0.209 \\
\hline Temperature $\left({ }^{\circ} \mathrm{C}\right)$, Median (Range) & $37.5(36.2-40)$ & 37.2(36.4-38.9) & -0.163 & 0.87 \\
\hline $\begin{array}{l}\text { Time from symptom onset to admission (days), } \\
\text { Median (Range) }\end{array}$ & $5(1-19)$ & $4(1-12)$ & -0.131 & 0.896 \\
\hline
\end{tabular}

(*): Significant, (SD): Standard deviation, (DM): Diabetes mellitus, (HTN): Hypertension, (IHD): Ischemic heart disease, (CKD): Chronic kidney disease, (SBP): Systolic blood pressure, (DBP): Diastolic blood pressure.

In addition, significant differences wereobserved between the studied groups as regards laboratory data. D-dimer, CRP, ferritin, LDH, serum creatinine, and blood urea were significantly higher in group 2 than in group 1, but total protein and eGFR were significantly lower in group 2 than in group 1 as shown in table (2).

Table (2): Comparison between the two groups regarding laboratory data

\begin{tabular}{|c|c|c|c|c|}
\hline Variable & Group 1 & Group 2 & Statistics & $P$ \\
\hline WBC $\left(\times 10^{3} / \mathrm{mm}^{3}\right)$, Median & 8.65 & 11.3 & -1.58 & 0.11 \\
\hline Lymphocyte $\left(\mathrm{x} 10^{3} / \mathrm{mm}^{3}\right)$, Median & 1.05 & 1 & -0.05 & 0.96 \\
\hline Hemoglobin (gm/dL), Median & 13.09 & 12.6 & -0.092 & 0.927 \\
\hline Platelets $\left(\times 10^{3} / \mathrm{mm}^{3}\right)$, Median & 233 & 200 & -1.36 & 0.17 \\
\hline Total protein $(\mathrm{g} / \mathrm{dL})$, Median & 7.1 & 6.3 & -2.705 & $0.007 * *$ \\
\hline Serum albumin $(\mathrm{g} / \mathrm{dL})$, Mean \pm SD & $3.55 \pm 0.57$ & $3.24 \pm 0.56$ & 1.941 & 0.057 \\
\hline Serum calcium $(\mathrm{mg} / \mathrm{dL})$, Median & 9 & 9.02 & -0.184 & 0.854 \\
\hline Serum phosphorus (mg/dL), Median & 3.29 & 3.34 & -0.606 & 0.544 \\
\hline Serum alkaline phosphatase (U/L), Median & 79 & 80.4 & -0.844 & 0.399 \\
\hline CRP (mg/L), Median & 125 & 223 & -2.08 & $0.04 *$ \\
\hline Serum D-dimer (mg/L), Median & 1.1 & 3.4 & -2.954 & $0.003 * *$ \\
\hline Serum ferritin (ng/mL), Median & 753 & 1348 & -2.93 & $0.003 * *$ \\
\hline Serum LDH, (IU/L), Median & 433 & 616 & -2.716 & $0.007 * *$ \\
\hline Serum troponin $(\mathrm{ng} / \mathrm{mL})$, Median & 0.12 & 0.03 & -1.783 & 0.075 \\
\hline Creatinine (mg/dL), Median & 0.86 & 1.13 & -3.942 & $<0.001 * *$ \\
\hline Blood urea $(\mathrm{mg} / \mathrm{dL})$, Median & 32.4 & 46.9 & -3.543 & $<0.001 * *$ \\
\hline eGFR $\left(\mathrm{ml} / \mathrm{m} / 1.73 \mathrm{~m}^{2}\right)$, Mean \pm SD & $87.7 \pm 8$ & $49.84 \pm 5$ & 4.61 & $<0.001 * *$ \\
\hline
\end{tabular}

$(*)$ : Significant, $(* *)$ : Highly significant, (SD): Standard deviation, (WBC): White blood cells, (CRP): C-reactive protein, (eGFR): Estimated glomerular filtration rate, (LDH): Lactate dehydrogenase. 
Additionally, analysis of predictors of AKI in all studied patients on univariate analysis showed that the significant predictors were older age, lower DBP, higher WBC count, higher CRP, higher serum ferritin, higher serum LDH, higher serum creatinine, higher blood urea, and lower eGFR as demonstrated in table (3).

Table (3): Univariate logistic regression analysis of predictors of AKI in all studied patients

\begin{tabular}{|l|c|c|c|c|c|}
\hline Variable & $\beta$ & SE & OR & $95 \%$ CI & $P$ \\
\hline Age & 0.04 & 0.02 & 1.037 & $(1-1.076)$ & $0.05^{*}$ \\
\hline Male & 0.47 & 0.60 & 1.604 & $(0.5-5.146)$ & 0.43 \\
\hline History of DM & -1.10 & 0.61 & 0.333 & $(0.102-1.091)$ & 0.07 \\
\hline History of HTN & -1.05 & 0.62 & 0.35 & $(0.104-1.173)$ & 0.09 \\
\hline History of IHD & -0.03 & 0.74 & 0.976 & $(0.231-4.125)$ & 0.97 \\
\hline History of CKD & -1.012 & 0.603 & 0.364 & $(0.112-1.186)$ & 0.093 \\
\hline SBP & -0.03 & 0.02 & 0.972 & $(0.942-1.004)$ & 0.09 \\
\hline DBP & -0.06 & 0.03 & 0.944 & $(0.896-0.996)$ & $0.04^{*}$ \\
\hline WBC & 0.10 & 0.05 & 1.107 & $(1.003-1.222)$ & $0.04^{*}$ \\
\hline Lymphocytes & 0.02 & 0.49 & 1.024 & $(0.396-2.651)$ & 0.96 \\
\hline Hemoglobin & -0.04 & 0.12 & 0.961 & $(0.759-1.217)$ & 0.74 \\
\hline Platelets & -0.01 & 0.00 & 0.996 & $(0.989-1.002)$ & 0.20 \\
\hline CRP & 0.01 & 0.00 & 1.005 & $(1.001-1.01)$ & $0.03^{*}$ \\
\hline Serum ferritin & 0.00 & 0.00 & 1.001 & $(1.000-1.002)$ & $0.01^{* *}$ \\
\hline Serum LDH & 0.00 & 0.00 & 1.003 & $(1.001-1.006)$ & $0.01^{* *}$ \\
\hline Serum D-dimer & 0.05 & 0.03 & 1.047 & $(0.99-1.108)$ & 0.11 \\
\hline Serum creatinine & 1.66 & 0.64 & 5.242 & $(1.485-18.503)$ & $0.01^{* *}$ \\
\hline Blood urea & 0.02 & 0.01 & 1.021 & $(1.005-1.038)$ & $0.01^{* *}$ \\
\hline eGFR & -0.05 & 0.01 & 0.951 & $(0.924-0.978)$ & $<0.001^{* *}$ \\
\hline Serum albumin & -0.46 & 0.39 & 0.634 & $(0.295-1.365)$ & 0.24 \\
\hline Total protein & -0.27 & 0.24 & 0.76 & $(0.479-1.207)$ & 0.25 \\
\hline Serum calcium & -0.84 & 0.60 & 0.431 & $(0.132-1.411)$ & 0.16 \\
\hline Serum phosphorus & 0.50 & 0.32 & 1.65 & $(0.884-3.082)$ & 0.12 \\
\hline $\begin{array}{l}\text { Serum alkaline } \\
\text { phosphatase }\end{array}$ & 0.01 & 0.01 & 1.013 & $(0.993-1.033)$ & 0.21 \\
\hline
\end{tabular}

(*): Significant, $(* *)$ : Highly significant, $(\mathrm{SE})$ : Standard error, $(\beta)$ : Regression coefficient, $(\mathrm{OR})$ : Odds ratio, (DM): Diabetes mellitus, (HTN): Hypertension, (IHD): Ischemic heart disease, (CKD): Chronic kidney disease, (SBP): Systolic blood pressure, (DBP): Diastolic blood pressure, (WBC's): White blood cells, (CRP): C-reactive protein, (LDH): Lactate dehydrogenase, (eGFR): Estimated glomerular filtration rate.

Furthermore, multivariate analysis showed that the significant predictors of AKI were lower DBP (OR: 0.899, 95\% CI: 0.813-0.993, P = 0.036), higher CRP (OR: 1.01, 95\% CI: 1.002-1.018, P = 0.017), higher serum LDH (OR: $1.005,95 \%$ CI: $1.001-1.008, \mathrm{P}=0.006)$ and lower eGFR (OR: $0.902,95 \% \mathrm{CI}: 0.842-0.966, \mathrm{P}=0.003)$ as represented in table (4).

Table (4): Multivariate logistic regression analysis of predictors of AKI in all studied patients

\begin{tabular}{|l|c|c|c|c|c|}
\hline Variable & $\beta$ & SE & OR & $95 \%$ CI & $P$ \\
\hline DBP & -0.107 & 0.051 & 0.899 & $(0.813-0.993)$ & $0.036^{*}$ \\
\hline CRP & 0.01 & 0.004 & 1.01 & $(1.002-1.018)$ & $0.017^{*}$ \\
\hline LDH & 0.005 & 0.002 & 1.005 & $(1.001-1.008)$ & $0.006^{* *}$ \\
\hline eGFR & -0.103 & 0.035 & 0.902 & $(0.842-0.966)$ & $0.003^{* *}$ \\
\hline
\end{tabular}

$(*)$ : Significant, $(* *)$ : Highly significant, (SE): Standard error, $(\beta)$ : Regression coefficient, $(\mathrm{OR})$ : Odds ratio, $(\mathrm{CKD})$ : Chronic kidney disease, (DBP): Diastolic blood pressure, (CRP): C-reactive protein, (LDH): Lactate dehydrogenase, (eGFR): Estimated glomerular filtration rate. 


\section{DISCUSSION}

Hospitalized patients are frequently affected by AKI (incidence $8 \%-22 \%$ ), which is linked to exaggerated morbidity and mortality ${ }^{(6)}$. A comparatively higher percentage of patients developed AKI in the current study $(22.7 \%)$. Recent studies have proposed the interaction of exaggerated inflammatory reaction, virusmediated injury, hypercoagulability, overactivity of renin-angiotensin system, and microangiopathy in severe infection with COVID-19, which increases the risk for multiple organ dysfunction ${ }^{(7)}$. Therefore, this research aimed to identify effects of COVID-19 infection on functions of the kidney and to identify predictors of AKI in COVID-19 infection.

This study included 66 participants. They were classified into group 1 (51 COVID-19 confirmed patients who did not develop AKI) and group 2 (15 COVID-19 confirmed patients who developed AKI). In comparison between the two groups as regards demographic and clinical data, subjects of group 2 were significantly older than subjects of group 1 . This is similar to the results obtained by Wang et al. ${ }^{(8)}$ but against Zahid et al. ${ }^{\left({ }^{(9)}\right.}$, which could be due to that older patients are more susceptible to severe infection by COVID-19 ${ }^{(\mathbf{1 0})}$. Additionally, group 2 had significantly lower DBP at the time of presentation than group 1. This agrees with results obtained by Zhunuspekova et al. ${ }^{\text {(11) }}$ who reported that lower DBP causes inadequate organ perfusion and renal ischemia thus AKI develops ${ }^{(\mathbf{1 2})}$.

In the comparison between the two groups regarding laboratory data, acute inflammatory markers, including serum D-dimer, serum CRP, serum ferritin and serum $\mathrm{LDH}$, were higher in group 2 than in group 1. These results agree with those reported by Hachim et al. ${ }^{(13)}$. The elevation of inflammatory markers may be mediated by cytokine release syndrome, which is an inflammatory immune reaction that results in multiple organ failure in severe Covid-19 ${ }^{\mathbf{( 1 4 )}}$. Interleukin (IL)-6 activates the liver to generate CRP and fibrinogen ${ }^{(\mathbf{1 5})}$. Additionally, plasma IL-6 is correlated with the high levels of LDH and ferritin resulting in increased synthesis of IL-18 by macrophages ${ }^{(16)}$. Then IL-18 enhances the cytotoxic activity of CD8+ $\mathrm{T}$ cells causing serious pulmonary damage ${ }^{(\mathbf{1 7})}$. In addition, group 2 had lower serum protein levels than group 1 . Generally, hypoproteinemia is a part of malnutrition that occurs in patients with AKI as a result of poor diet, increased protein catabolism, oxidative stress, inflammatory cytokine generation, insulin resistance, salt and water retention, metabolic acidosis and associated chronic debilitating diseases ${ }^{(\mathbf{1 8})}$.

In comparison of renal function at the time of presentation of the patients to the hospital between the two groups, group 2 had significantly higher serum creatinine, higher blood urea and lower eGFR. This is similar to results obtained by Hachim et al. ${ }^{(13)}$. This might be because CKD patients suffer from reduced ability to handle stress such as hypotension or nephrotoxic medications and have significantly lower renal reserve. However, that is not well comprehended how precisely pathophysiologically CKD is linked to AKI ${ }^{(19)}$.

On the univariate analysis of predictors of AKI in all studied patients, the risk factors were older age, lower DBP, higher WBC count, higher CRP, higher serum ferritin, higher serum $\mathrm{LDH}$, higher serum creatinine, higher blood urea, and lower eGFR. Then, significant predictors were analyzed by multivariate analysis and the significant risk factors for AKI in COVID-19 confirmed patients were lower DBP, higher CRP, higher serum LDH, and lower eGFR. Lower DBP was one of the significant risk factors associated with AKI in the studied patients as it is a part of septic shock that occurs in severe cases of COVID-19 patients. Septic shock causes an extensive capillary leukocytic invasion, apoptosis and infrequent thrombi in the kidneys ${ }^{(20)}$. Additionally, both higher CRP and higher serum LDH represented important risk factors correlated with $\mathrm{AKI}$ in the studied patients. CRP has been shown to lower the generation of nitric oxide and hinder the defensiveness of antioxidants, resulting in endothelial dysfunction and reduced responsiveness to renal vasodilators ${ }^{(21)}$. Also, high LDH levels can lead to AKI as it causes oxidative stress, inflammatory reactions, triggering of proapoptotic proteins, vasospasm, and blockage of renal tubules ${ }^{(22)}$. Finally, the lower baseline eGFR was a highly significant risk factor for the renal injury due to the lessened capacity of the kidneys to manipulate stress in severe COVID-19 infection.

\section{CONCLUSION}

In conclusion, COVID-19 infection represents a worldwide health problem and AKI is one of its most significant sequelae. AKI occurred in $22.7 \%$ of hospitalized patients with confirmed COVID-19 infection in this study. The most important risk factors of the occurrence of AKI in COVID-19 patients were lower DBP, higher CRP level, higher serum LDH level, and lower baseline eGFR.

Funding: None. Author funded.

\section{Conflict of interest: None.}

\section{ACKNOWLEDGMENT}

I acknowledge the staff employed in the Internal Medicine Department of the hospital and the participants of the research who without their cooperation, this study would not have been accomplished. 


\section{REFERENCES}

1. Hui D I, Azhar E, Madani T et al. (2020): The continuing 2019-nCoV epidemic threat of novel coronaviruses to global health - The latest 2019 novel coronavirus outbreak in Wuhan, China. Int J Infect Dis., 91: 264-266.

2. Huang C, Wang Y, Li X et al. (2020): Clinical features of patients infected with 2019 novel coronavirus in Wuhan, China. The Lancet, 395: 497-506.

3. Henry B, de Oliveira M, Benoit $S$ et al. (2020): Hematologic, biochemical and immune biomarker abnormalities associated with severe illness and mortality in coronavirus disease 2019 (COVID-19): a metaanalysis. Clin Chem Lab Med., 58: 1021-1028.

4. Collado S, Arenas M, Barbosa F et al. (2020): COVID19 in Grade 4-5 Chronic Kidney Disease Patients. Kidney Blood Press Res., 45: 768-774.

5. Khwaja A (2012): KDIGO Clinical Practice Guidelines for Acute Kidney Injury. Nephron Clin Pract., 120: c179c184.

6. Khadzhynov D, Schmidt D, Hardt J et al. (2019): The Incidence of Acute Kidney Injury and Associated Hospital Mortality. Dtsch Arztebl Int., 116: 397-404.

7. Batlle D, Soler M, Sparks M et al. (2020): Acute Kidney Injury in COVID-19: Emerging Evidence of a Distinct Pathophysiology. J Am Soc Nephrol., 31: 1380-1383.

8. Wang F, Ran L, Qian C et al. (2020): Epidemiology and Outcomes of Acute Kidney Injury in COVID-19 Patients with Acute Respiratory Distress Syndrome: A Multicenter Retrospective Study. Blood Purif., 12: 1-7.

9. Zahid U, Ramachandran P, Spitalewitz S et al. (2020): Acute Kidney Injury in COVID-19 Patients: An Inner City Hospital Experience and Policy Implications. Am J Nephrol., 51: 786-796.

10. Clark A, Jit M, Warren-Gash C et al. (2020): Global, regional, and national estimates of the population at increased risk of severe COVID-19 due to underlying health conditions in 2020: a modelling study. Lancet Glob Health, 8: e1003-e1017.

11. Zhunuspekova A, Mansurova J, Karazhanova L (2021): Independent predictors of acute kidney injury in patients with acute coronary syndrome after percutaneous coronary intervention. Plos one, 16: e0247304.

12. Sato R, Luthe S, Nasu M (2017): Blood pressure and acute kidney injury. Crit Care, 21: 1-2.

13. Hachim brahim Y, Hachim M, Naeem K et al. (2021): Kidney Dysfunction among COVID-19 Patients in the United Arab Emirates. Oman Med J., 36: e221-e221.

14. Ciceri F, Beretta L, Scandroglio A et al. (2020): Microvascular COVID-19 lung vessels obstructive thromboinflammatory syndrome (MicroCLOTS): an atypical acute respiratory distress syndrome working hypothesis. Crit Care Resusc., 22: 95-97.

15. Zhang T, Sun L, Feng R (2020): Comparison of clinical and pathological features between severe acute respiratory syndrome and coronavirus disease 2019. Zhonghua Jie He He Hu Xi Za Zhi, 43: 496-502.

16. Budu-Aggrey A, Brumpton B, Tyrrell J et al. (2019): Evidence of a causal relationship between body mass index and psoriasis: A mendelian randomization study. PLoS Med., 16: e1002739.

17. Sattar N, McInnes I, McMurray J (2020): Obesity a Risk Factor for Severe COVID-19 Infection: Multiple Potential Mechanisms. Circulation, 142: 4-6.

18. Li G, Chen X, Zhang Y et al. (2010): Malnutrition and inflammation in acute kidney injury due to earthquakerelated crush syndrome. BMC Nephrol., 11: 1-6.

19. Hsu R, Hsu C (2016): The Role of Acute Kidney Injury in Chronic Kidney Disease. Semin Nephrol., 36: 283-292.

20. Lerolle $\mathrm{N}$, Nochy $\mathrm{D}$, Guérot $\mathrm{E}$ et al. (2010): Histopathology of septic shock induced acute kidney injury: apoptosis and leukocytic infiltration. Intensive Care Med., 36: 471-478.

21. Cosentino N, Genovese S, Campodonico J et al. (2019): High-Sensitivity C-Reactive Protein and Acute Kidney Injury in Patients with Acute Myocardial Infarction: A Prospective Observational Study. J Clin Med., 8: 2192.

22. Yin M, Jiang $\mathrm{N}$, Guo $\mathrm{L}$ et al. (2019): Oleuropein suppresses oxidative, inflammatory, and apoptotic responses following glycerol-induced acute kidney injury in rats. Life Sci., 232: 116634. 